\title{
Identification of intrinsically disorder regions in non-structural proteins of SARS-CoV-2: New insights into drug and vaccine resistance
}

\author{
Farah Anjum ${ }^{1} \cdot$ Taj Mohammad $^{2}$ (D) Purva Asrani ${ }^{3} \cdot$ Alaa Shafie $^{1} \cdot$ Shailza Singh $^{4} \cdot$ Dharmendra Kumar Yadav $^{5}$. \\ Vladimir N. Uversky ${ }^{6} \cdot$ Md Imtaiyaz Hassan $^{2}$ (D)
}

Received: 1 November 2021 / Accepted: 10 February 2022 / Published online: 24 February 2022

(c) The Author(s), under exclusive licence to Springer Science+Business Media, LLC, part of Springer Nature 2022

\begin{abstract}
The outbreak of severe acute respiratory syndrome coronavirus-2 (SARS-CoV-2) emerged in December 2019 and caused coronavirus disease 2019 (COVID-19), which causes pneumonia and severe acute respiratory distress syndrome. It is a highly infectious pathogen that promptly spread. Like other beta coronaviruses, SARS-CoV-2 encodes some non-structural proteins (NSPs), playing crucial roles in viral transcription and replication. NSPs likely have essential roles in viral pathogenesis by manipulating many cellular processes. We performed a sequence-based analysis of NSPs to get insights into their intrinsic disorders, and their functions in viral replication were annotated and discussed in detail. Here, we provide newer insights into the structurally disordered regions of SARS-CoV-2 NSPs. Our analysis reveals that the SARS-CoV-2 proteome has a chunk of the disordered region that might be responsible for increasing its virulence. In addition, mutations in these regions are presumably responsible for drug and vaccine resistance. These findings suggested that the structurally disordered regions of SARS-CoV-2 NSPs might be invulnerable in COVID-19.
\end{abstract}

Keywords SARS-CoV-2 · COVID-19 - Intrinsically disordered proteins · Vaccine development $\cdot$ Molecular pathogenesis . COVID-19 therapeutics

\section{Introduction}

Dharmendra Kumar Yadav

dharmendra30oct@gmail.com

Md Imtaiyaz Hassan

mihassan@jmi.ac.in

1 Department of Clinical Laboratory Sciences, College of Applied Medical Sciences, Taif University, P.O. Box 11099, Taif 21944, Saudi Arabia

2 Centre for Interdisciplinary Research in Basic Sciences, Jamia Millia Islamia, Jamia Nagar, New Delhi 110025, India

3 Department of Microbiology, University of Delhi, New Delhi 110021, India

4 National Centre for Cell Science, NCCS Complex, Ganeshkhind, SP, Pune University Campus, Pune 411007, India

5 College of Pharmacy, Gachon University of Medicine and Science, Hambakmoeiro, Yeonsu-gu, Incheon City 21924, South Korea

6 Department of Molecular Medicine and USF Health Byrd Alzheimer's Institute, Morsani College of Medicine, University of South Florida, Tampa, FL 33612, USA
Severe Acute Respiratory Syndrome-Coronavirus-2 (SARSCoV-2) causing coronavirus disease 2019 (COVID-2019) has been the leading cause of deaths [1]. Various ups and downs in the SARS-CoV-2 infection pattern have been accredited to the mutations in the structural proteins of the virus, especially spike (S) protein [2]. Presently, the total number of confirmed cases across the globe stands at $>350$ million, whereas 5.6 million people have died (assessed on 25th January 2022) [3]. Many nations have countersigned the rapid spread of SARS-CoV-2 infection in different waves after certain time intervals [4-6]. Scientists have cautioned in contradiction of the forthcoming peaks of the present waves and the coming of new waves, which are yet to come in many countries $[7,8]$. The severity of SARS-CoV-2 infection has led to the thorough lockdown in most parts of the world, leading to the physical and psychological impact on people [9-13].

SARS-CoV-2 has four capsid-forming structural proteins: spike protein (S) that assists in attaching the virus to the ACE receptor of host cells [14]; membrane protein (M) 
that forms viral membrane for encircling the mature viral particles [15]; nucleocapsid protein $(\mathrm{N})$ that creates a viral protein coat, i.e., nucleocapsid for surroundings the genetic material [16]; and envelope protein (E) that forms the envelope for assembling the virions [17]. The SARS-CoV-2 genome is more than $80 \%$ similar to a previous SARS-CoV strain that triggered an outbreak in 2003 [18, 19]. Consequently, it exhibits a similar replication process as witnessed in the earlier cases.

Apart from the capsid-forming structural proteins, the SARS-CoV-2 genome encodes many non-structural proteins (NSPs), playing significant roles in the replication and virus assembly [20, 21]. NSPs participate in SARS-CoV-2 pathogenesis by controlling early transcription regulation, transactivation, helicase activity, immunomodulation, and disputing the antiviral response [22-24]. A few essential functions of the SARS-CoV-2 NSPs are RNA-binding, transferase activity, ATP-binding, zinc-binding, endopeptidase activity, RNA-dependent RNA polymerase activity, exoribonuclease activity, and methyltransferase activity. They also participate in numerous biological processes such as transcription, replication, protein processing, and proteolysis [25].

Similar to other viral proteomes, the SARS-CoV-2 proteome has chunks of intrinsically disordered regions. It is important to explore the disorder status in NSPs to better understand the roles of these proteins in the virulence. We performed a sequence-based analysis on all NSPs specific to SARS-CoV-2 to get insights into their intrinsic disorder status to look for functions of the disordered regions and their roles in viral replication. Here, we provide a computational structural disorder landscape of the SARS-CoV-2 NSPs. The available sequence data for SARS-CoV-2 NSPs were analyzed to evaluate their intrinsic disorder in light of their biological activity relationships.

\section{Material and methods}

The sequence data of the SARS-CoV-2 NSPs were taken from the UniProt [26] (UniProtKB: P0DTD1) and GenBank ${ }^{\circledR}$ [27]. To explore the intrinsically disordered regions in SARS-CoV-2 NSPs, multiple bioinformatics tools such as PONDR-FIT ${ }^{\circledR}$ (Predictor of Natural Disordered Regions), VLXT, VL3, VSL2B [28] and IUPred2A web servers (IUP2(S) and IUP2 (L)) [29] along with $\sigma(\mathrm{MDP})$. These predictors classify intrinsically disordered regions in a protein by predicting the residues which do not show the tendency to form a tertiary structure in the native conditions. The predictors consider a residue intrinsically disordered when it scores $>0.5$ and flexible to the residue with a score of $0.2-0.5$. During the analyses, the residues in each NSP were renumbered from 1. However, their original positions are shown in the titles of the figures (Figs. 1, 2, 3, 4). The analysis provides an insight into the intrinsically disordered regions in SARS-CoV-2 NSPs that may be valuable for understanding the SARS-CoV-2 virulence $[25,30]$.
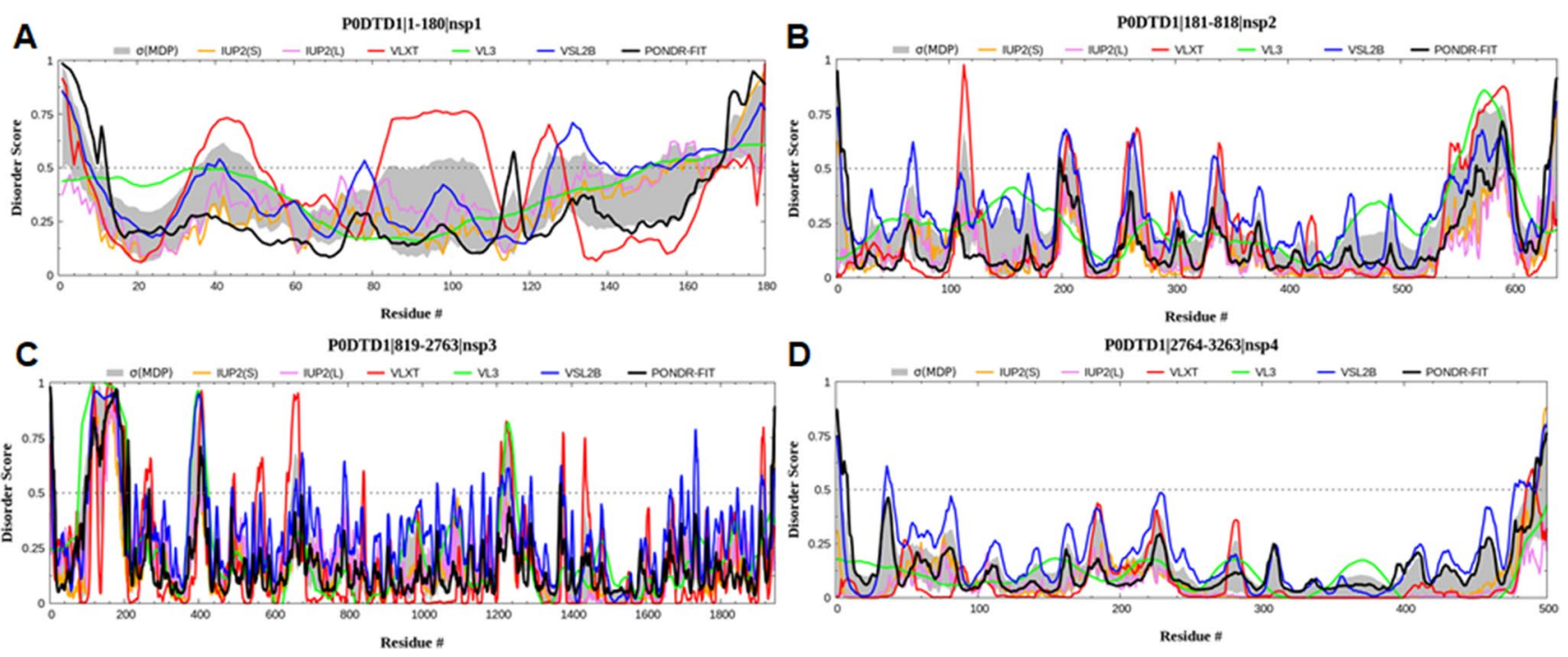

Fig. 1 Graph showing the disordering tendency of each residue in SARS-CoV-2 a NSP1, b NSP2, c NSP3, and d NSP4. The middle line is the threshold value of the PONDR score, i.e., 0.5. The residues

in each NSP were renumbered from 1; however, their original positions are shown in the titles of each panel 

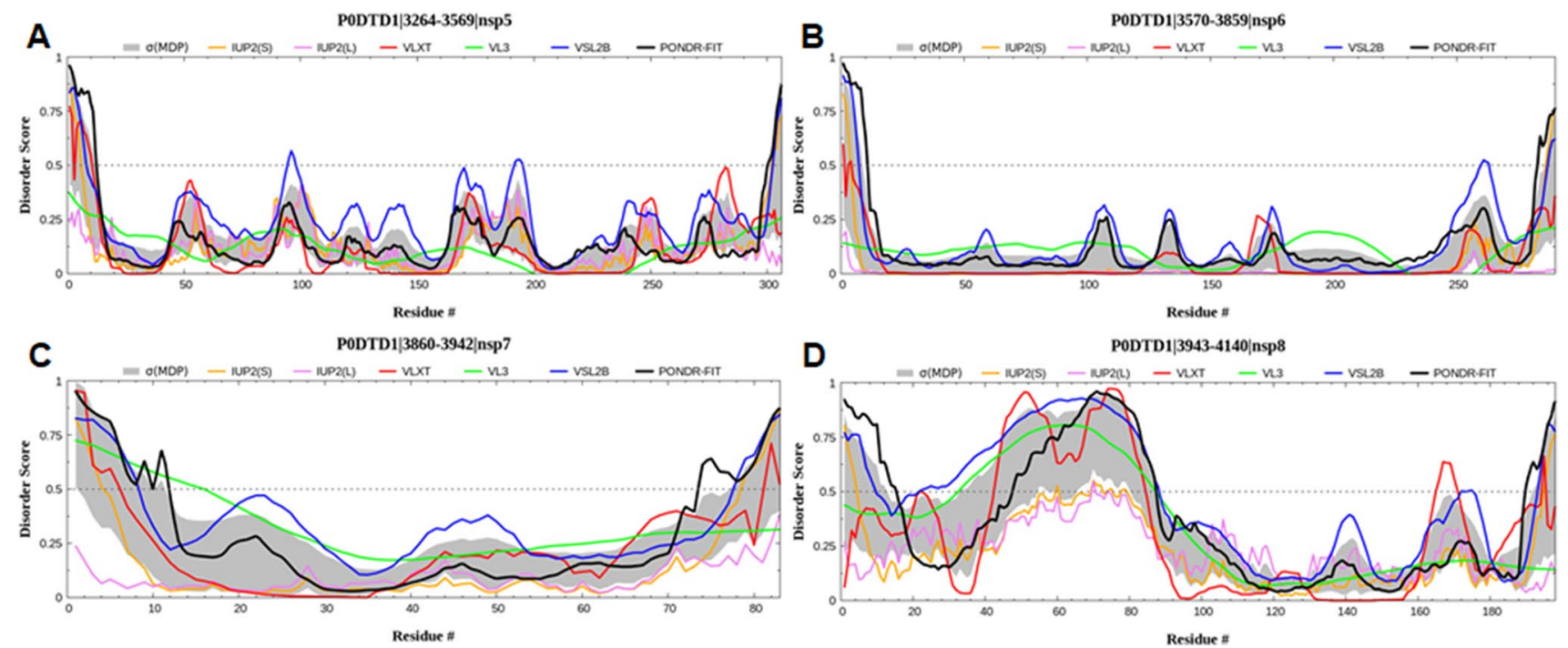

Fig. 2 Graph showing the disordering tendency of each residue in SARS-CoV-2 a NSP5, b NSP6, $\mathbf{c}$ NSP7, and $\mathbf{d}$ NSP8. The middle line is the threshold value of the PONDR score, i.e., 0.5

\section{Results and discussion}

Two-thirds of the SARS-CoV-2 genome comprises ORF $1 \mathrm{a} / \mathrm{b}$ genes, leading to the production of two different replicase polyproteins, pp1a and pp1b, respectively. These polyproteins undergo further processing by proteases to produce 16 NSPs that assist in replication, transcription, assembly, and packaging of the virion particles [31]. These proteins regulate virus functions, and targeting these proteins is therefore ideal for devising treatment against the coronavirus.

\section{Non-structural protein 1 (NSP1)}

A papain-like proteinase (PLpro) cleaves replicase polyprotein of coronaviruses to yield an $\mathrm{N}$-terminal product called NSP1 [32]. The main functions associated with the NSP1 include degradation of viral mRNA, blocking of host cell translation process, and inhibition of the host's innate immune response to initiate a successful viral replication [33-35]. The viral evasion successfully suppresses the host genes, allowing the virus to control its immune system [36, 37]. NSP1 blocks translation by binding to the 40S subunit of rRNA, thus preventing the entry of host mRNA for subsequent translation [38]. This binding is initiated by the NSP1 carboxyl-terminal domain of SARS-CoV-2 [39]. Earlier studies have demonstrated that the deletion of the NSP1 gene in infectious virus particles resulted in the inability of the virus to infect the culture cells [40]. Mutations in the ORF1a polyprotein that prevented the release of NSP1 resulted in the limited viability of the virus [41].
In general, NSP1 consists of an $\alpha$-helix located at either side of seven stranded $\beta$-barrel with two $3_{10}$ helices positioned across one side of $\beta$-barrel. Clark et al. reported the sequence and structural similarities of NSP1 from SARSCoV-1 and SARS-CoV-2. However, minor differences were observed, which may be responsible for the difference in their viral pathogenic life cycles [42]. Firstly, an extra $\beta$-strand and $3_{10}$ helices were found in SARS-CoV-2. Secondly, an increased polarity between the amino acids and the globular domain resulted in alternative conformations of major loops in SARS-CoV-2. Thirdly, the differences in the amino acids showed different electrostatic surface potentials. These differences might have defined altered SARS-CoV-2 behaviour in terms of pathogenicity and infectivity. Very few structural studies have been done on NSP1, but it seems like a potential area to be explored in the coming time to identify its ability to be used as an antiviral drug treatment [42-47].

In the analysis of the intrinsic disorder's predisposition, the generated graph showed the disordering tendency of each residue in NSP1, where higher values correspond to a higher probability of being disordered (Fig. 1a). The graph indicated that NSP1 has many intrinsically disordered regions lacking well-defined structures in native circumstances. The region amino acid residues $25-50,80-110$ and $120-140$ of NSP1 showed a higher tendency of disorder as predicted by one or more predictors.

\section{Non-structural protein 2 (NSP2)}

NSP2 plays a significant role in modifying the host's environment, making it more suitable for viral needs. This protein is also involved in misbalancing the host's intracellular 

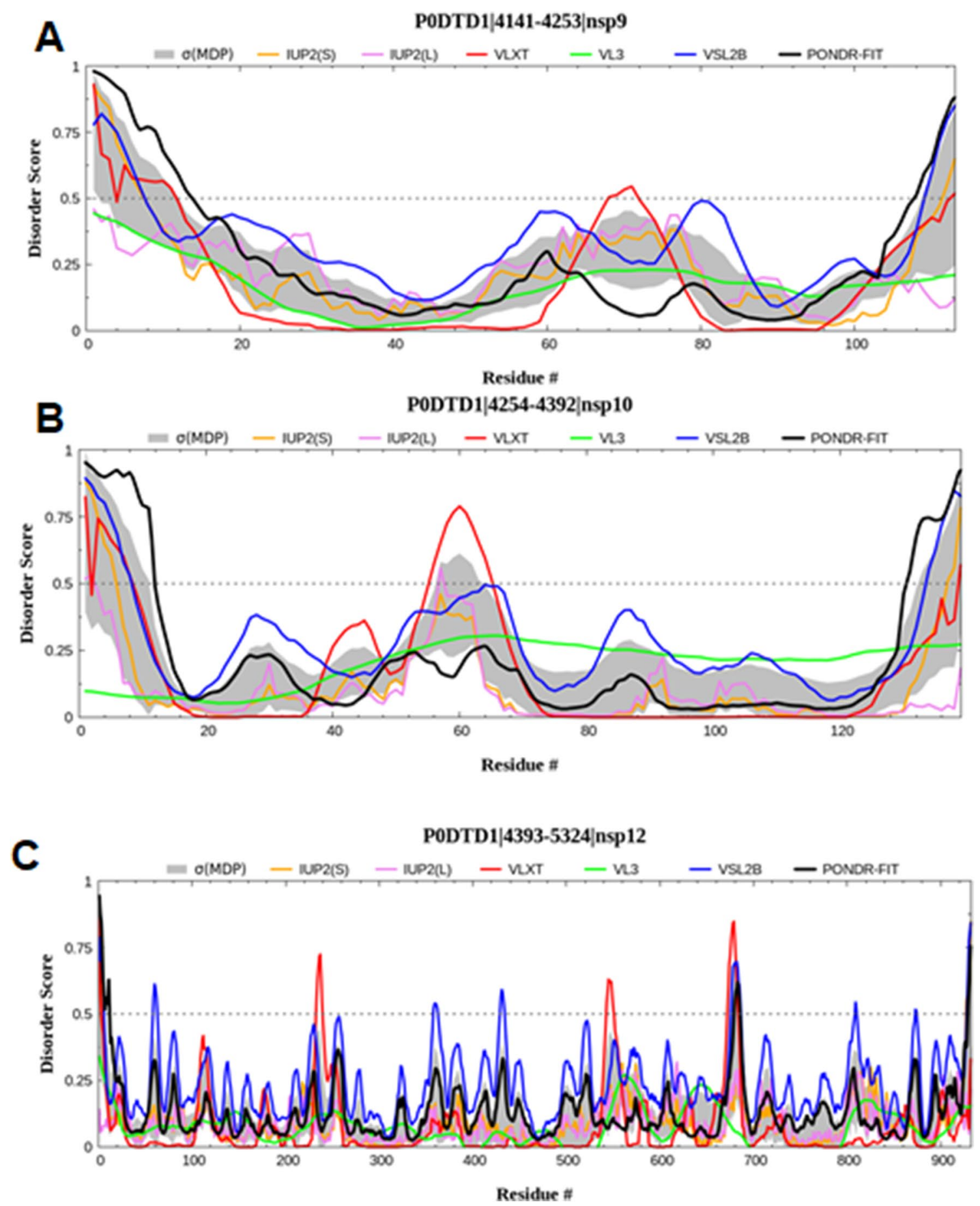

Fig. 3 Graph showing the disordering tendency of each residue in SARS-CoV-2 a NSP9, b NSP10, and $\mathbf{c}$ NSP12. The middle line is the threshold value of the PONDR score, i.e., 0.5

signaling pathways, though the precise mechanism of this function is not yet known. NSP2 possesses variability among different strains of coronaviruses [48]. Compared to batSARS like coronavirus, a polar amino acid was found at position 321 of the NSP2 protein compared to SARS-CoV-2, which contained a polar glutamine residue. The stability of this protein may be due to this change in polarity, allowing it to make hydrogen bonds, and due to the changes in the side chain length and interactions. A stabilizing mutation within the endosome-associated protein-like domain of NSP-2 was found in SARS-CoV-2, suggesting their highly contagious nature [49]. 

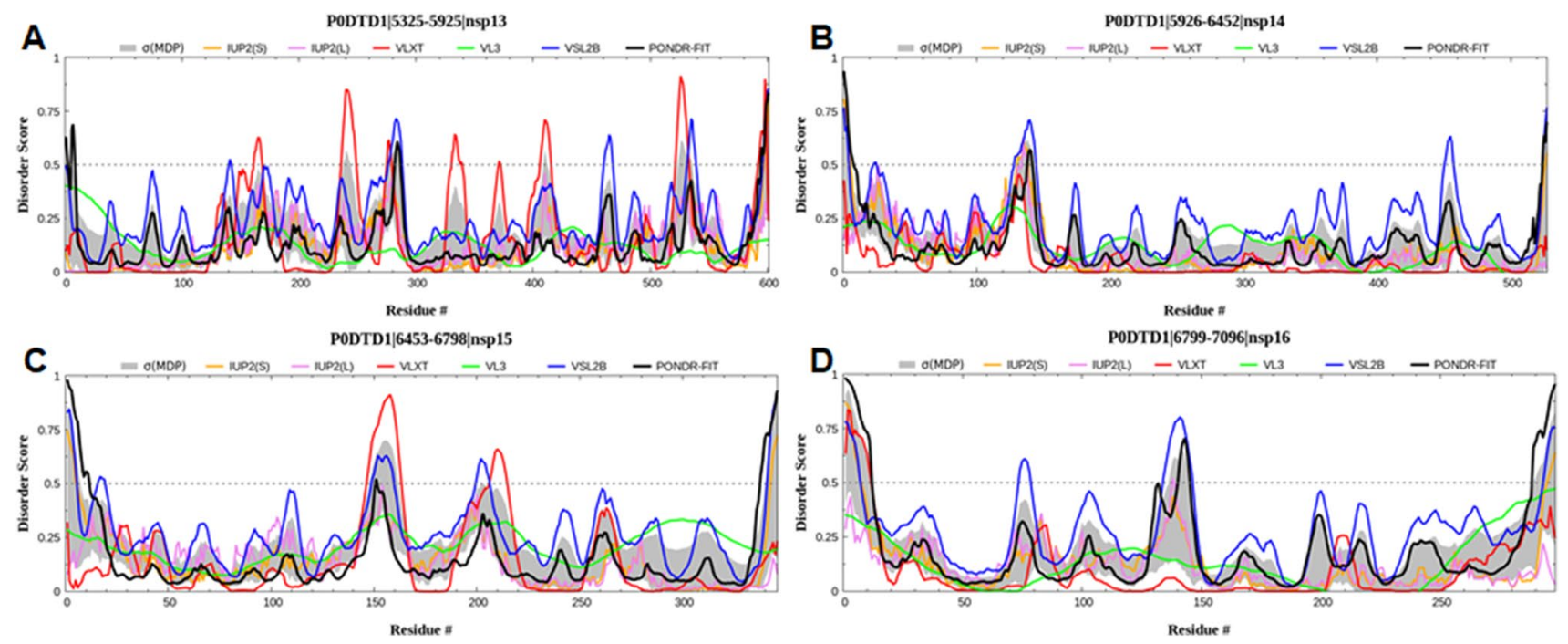

Fig. 4 Graph showing the disordering tendency of each residue in SARS-CoV-2 a NSP13, b NSP14, $\mathbf{c}$ NSP15 and d NSP16. The middle line is the threshold value of the PONDR score, i.e., 0.5

The interactors of coronavirus NSP2 proteins were identified, such as prohibitin 1 and prohibitin 2 having a profound role in mitochondrial biogenesis [50]. Evaluation of various other interactors by Davies et al. revealed their presence in the endoplasmic reticulum (ER) and mitochondrial membrane, suggesting the involvement of NSP2 in the regulation of $\mathrm{ER} \mathrm{Ca}^{2+}$ ion signaling [48]. NSP2 interacts with NSP3 and NSP4 to mediate different functions in promoting the viral infectious life cycle [51]. The complex ERLIN1/2 was found to interact with NSP2 and NSP4 present in both SARS-CoV and SARS-CoV-2 but absent in bat-like coronavirus. The interaction of these two NSP proteins with this complex might regulate $\mathrm{ER}^{2+} \mathrm{Ca}^{2+}$ signaling and the associated host responses related to these signaling cascades. NSP2 interaction with NSP3 is involved in the formation of proteases which cleaves ORF1ab [52]. More significant studies are required in this direction to identify their role in imparting viral pathogenesis fully.

The intrinsic disorder profile indicated that the NSP2 has several intrinsically disordered regions that lack a stable structure under physiologic conditions. The regions spanning residues $260-300,370-410,430-470$, and $730-780$ of NSP2 showed a higher tendency of disorder as predicted by all four predictors (Fig. 1b).

\section{Non-structural protein 3 (NSP3)}

NSP3 is a multi-pass transmembrane protein consisting of $\sim 1945$ amino acid residues. It is primarily associated with translating viral mRNA transcripts and inhibiting protein synthesis in the host $[53,54]$. Apart from this, it is also involved in delegating and deubiquitinating activities [55].
An interaction of NSP2 and NSP3 mediates cleavage of ORF1ab by coding for viral proteases. Such processing of polyprotein releases NSP1, NSP2, and NSP3. NSP3 also interacts with NSP4 where they, together in complex with other proteins, are involved in structural membrane rearrangement, facilitating viral replication [56].

The NSP3 structure consists of different functional domains. These domains include a SARS unique domain (SUD) having N-terminal, middle, and C-terminal subdomain $[57,58]$. In addition to this, an RNA binding domain facilitates the viral protein interaction with hosts rRNA [59], and the papain-like protease (PL-PRO) domain allows to code for proteases that regulate the full viral activity [60]. The intrinsic disorder graph showed that NSP3 has multiple intrinsically disordered regions distributed throughout the protein (Fig. 1c).

\section{Non-structural protein 4 (NSP4)}

NSP4 plays a key role in replicative structural assembly for coronavirus replication associated with the NSP3 protein [61]. The loss of the NSP3-NSP4 complex was associated with abolished viral replication [56]. NSP4 maintains interactions with many other proteins and cofactors to bring about other functions. These interactions are unique to different members of coronaviruses, such as interaction with members of the E3 ubiquitin ligase family in SARS-CoV and interactions with factors associated with ER homeostasis in SARS-CoV-2 [48]. Other commonly observed interactions of NSP4 were observed with proteins related to unfolded protein response (UPR) (TMEM33), ER-phagy (CCPG1), and machinery associated with $\mathrm{N}$-liked glycosylation 
(STT3B, MAGT1, CANX, DDOST). These signaling factors mediate an antiviral innate immune response (RNF5, an E3 ubiquitin ligase present in ER) [62, 63] and Vitamin K reducing complex (VKORC1), which is required by coagulation, causing proteins as a cofactor [64]. Like NSP2, these interactors are localized in the membranes of mitochondria and ER, regulating their role in mediating major functions related to the contact site like calcium homeostasis, biogenesis and cell organization, transport, and various other metabolic processes. Some of the NSP4 interactors were also found to have a role in cell communication, cell differentiation, and cell death [48]. The intrinsic disorder analysis suggested that the NSP4 doesn't have many disordered regions (Fig. 1d).

\section{Non-structural protein 5 (NSP5)/3C-like proteinase (3CLpro)}

NSP5, a $\sim 305$ amino acid long protein, is responsible for the maturation of other NSPs in the coronaviruses [65]. NSP5 is a cysteine protease that is automatically cleaved from the polyprotein to produce a mature enzyme, cleaving the polyprotein at 11 different locations to yield NSP4-NSP16 [66]. NSP5 is also referred to as $3 \mathrm{CL}^{\text {pro }}$, which has three distinct domains I-III, where domain II and domain III are connected via a loop consisting of a long amino acid stretch from 185 to 200 residues. The active site of this protein is in the form of a catalytic dyad (CysHis) located within domain I and domain II [67]. The catalytic residues (Cys145 and His41) and 3-domain structure are conserved among the NSP5, providing them the ability to mediate the viral activity by acting as a protease in viral maturation [68]. The intrinsic disorders graph indicated that the NSP5 does not have many disordered regions except at around residues 3360-3370 and 3530-3550 (Fig. 2a).

\section{Non-structural protein 6 (NSP6)}

NSP6 is a 290 amino acid long, a multi-pass membrane protein that induces the formation of double-membrane vesicles (DMV) in the host [69]. NSP6, along with NSP3 and NSP4, plays an important role in the coronavirus replication by forming a part of the replication/transcription complex (RTC) [70]. Several other NSPs are also involved in this complex, each assigned specific roles. Once NSP6 is inserted into the ER [71], it complexes with NSP3 and NSP4 for the formation of DMV during assembly of RTC [69].

NSP6 is $34 \mathrm{kDa}$ in size and consists of a C-terminal domain and six transmembrane helices [72]. The outer surface of NSP6 marks the presence of phenylalanine residues, providing higher binding affinity and stability between NSP6 and ER membrane [73]. This protein is commonly present in both $\alpha$ and $\beta$ coronaviruses, and its location is central to
ER, where it assists in forming autophagosomes [74]. This binding reduces the transfer of viral factors to lysosomes and thus promotes coronavirus replication [75]. Therefore, the expansion of the autophagosome is also compromised either by starvation or through chemical inhibition of mTOR signaling [76, 77]. NSP6 sends immunomodulatory proteins synthesized by the ER to autophagosomes for their destruction, a process that modifies the adaptive immune response of the host. In addition, NSP6 interaction with the sigma factor helps their participation in ER stress response [78]. The intrinsic disorder graph indicated that the NSP6 does not have many disordered regions, except at the end of its C-terminal region (Fig. 2b).

\section{Non-structural protein 7 (NSP7)}

NSP7 is a small protein of 83 amino acid residues in length. In association with NSP8, this protein acts as a cofactor for the activity of NSP12, which is RNA-dependent RNA polymerase (RdRp) required for viral transcription [79]. These three NSP forms a trimeric RdRp-NSP7-NSP8 super-complex [80], the basic minimal machinery required for nucleotide polymerization [79]. Mutations in NSP7 or NSP8 are associated with mutations of NSP12, and they might therefore show altered super-complex formation affecting the viral replication, infectivity, and pathogenicity [81]. Since NSP7 has less significance when unbound, few studies have been conducted on its structure. More studies on the supercomplex formation and its structure have been performed, where targeting one NSP could inhibit the process of viral replication, and transcription is majorly studied. The intrinsic disorder graph suggested that NSP7 does not have any disordered regions (Fig. 2c).

\section{Non-structural protein 8 (NSP8)}

NSP7, NSP8 forms a hexadecamer and acts as a cofactor for NSP12 activity required for viral replication. NSP8 is made of $\sim 198$ amino acid residues. Mutations of NSP8 were linked to the altered RNA synthesis in SARS-CoV-2 [82]. This protein may possess RNA processivity or RNA primase function [83]. NSP8 also interacts with NSP9 and is involved in replicating RNA virulence and promoting the virulence properties of SARS-CoV-2 [84]. More studies are warranted in assessing the impact of mutations on NSP8 linked to the RdRp-NSP7-NSP8 super-complex. Detailed understanding of the NSP8 structure is also necessary, as fewer studies have been performed to date. The intrinsic disorder graph showed that NSP8 has a chunk of intrinsically disordered regions distributed between residues 3960-4030 and 4110-4120 (Fig. 2d). 


\section{Non-structural protein 9 (NSP9)}

NSP9 consists of $\sim 113$ amino acid residues in length and primarily functions as a dimeric ssRNA-binding protein involved in viral replication [82]. The sequence of NSP9 is conserved among $\beta$-coronaviruses, especially among SARS-CoV and SARS-CoV-2, sharing almost 97\% homology [85]. NSP9 colocalizes with various NSPs and forms an important part of the replication machinery of coronaviruses and is therefore directly involved in their growth [86]. Studies have revealed that deletions in the NSP9 gene are marked by impaired synthesis of RNA and viral infectivity [84]. Protein-nucleic acid interactions are facilitated by multimerization of NSP9 [87]. During NSP9 dimerization, the two $\alpha$-helices bind to the GXXXG motif, an essential step for coronavirus replication [84].

In general, the protomer of NSP9 consists of seven $\beta$-strands surrounded by $\mathrm{N}$-terminal and $\mathrm{C}$-terminal $\alpha$-helices. The protein core is made up of $\beta$-barrel comprising two orthogonally packed antiparallel $\beta$-sheets. One sheet consists of $\beta_{1}-\beta_{3}$ strands along with half $\beta_{7}$-strand, while the other sheet is made up of $\beta_{4}$ and $\beta_{5-}$ strands. This $\beta$-barrel is extended with the help of $\beta$-hairpin consisting of $\beta_{6}$ and $\beta_{7}$-strands adjoining $\alpha$-helix. The barrel sheets are linked to $\mathrm{N}$-terminal $\beta$-strand and $\mathrm{C}$-terminal $\alpha$-helix via elongated loops [88]. Such NSP9 protomer folding is similar to the OB-fold, a structural motif that recognizes nucleic acids [89].

The structural analysis of NSP9 in SARS-CoV-2 revealed a horseshoe-like tetramer structure, which assists in its oligomerization and binding to the virus's nucleic acid during the replication process. The stabilization of this protein occurs via the presence of two contact surfaces. The first interaction is a parallel association between $\alpha$-helix at the $\mathrm{C}$-terminal region with $\mathrm{N}$-terminal loop ( $\alpha$-helix interface) [90]. It then involves the antiparallel interactions between two $\beta$-strands at both protomers of NSP9 ( $\beta$-sheet interface) [86], bringing the two barrels together for function and stabilization. In SARS-CoV-2, the sheet interface accounts for more stability in the tetrameric structure of NSP9 than in SARS-CoV. Breaking of the dimeric structure of NSP9 affects their stability and, in turn, prevents RNA synthesis and viral growth [91]. The intrinsic disorders graph suggested that the NSP9 has no disordered regions (Fig. 3a).

\section{Non-structural protein 10 (NSP10)}

NSP10 is a $\sim 130$ amino acid residues long scaffold protein involved in stimulating NSP14 and NSP16. NSP14 possesses two distinct domains that mediate different functions. N-terminal consists of $3^{\prime}-5^{\prime}$ exoribonuclease activity (ExoN), and C-terminal possesses N7-methyltransferase (N7-MTase) activity [82]. NSP10 binding to ExoN of
NSP14 causes its stimulation; however, N7-MTase remains unaffected with this binding [92]. NSP10 also stimulates the activity of 2'-O-methyltransferase (2'-O-MTase) in NSP16 [93]. Therefore, NSP10 serves as an essential protein for activating the methylation and capping machinery for viral mRNA in association with NSP14 and NSP16 [94]. This protein is exclusively found in viruses and not in prokaryotes or eukaryotes.

The structural features of NSP10 consist of a long loop which connects two antiparallel $\alpha$-helices (H1 and H2) with a $\beta_{1}$-strand, followed by a sheet formation involving $\beta_{2}$ and $\beta_{3}$-strands [95]. Two zinc finger motifs are present in the NSP10 structure. In the first case, the $\beta$-sheet is folded into two helices named $\mathrm{H} 3$ and $\mathrm{H} 4$, containing the zinc finger comprised three cysteines and one histidine to coordinate the zinc ion. Then a small helical turn is present after $\beta_{3}$ adjoining $\alpha$-helix H6. The two strands $\beta_{4}$ and $\beta_{5}$ interrupt the long $\mathrm{C}$-terminal region, which harbors the second zinc finger motif composed of only four cysteine side chains as required to stabilize this non-classical zinc finger protein $[95,96]$. NSP10 of SARS-CoV-2 exhibited high structural similarity to NSP10 of SARS-CoV [95]. The intrinsic disorder graph indicated that NSP10 does not have many disordered regions, except at the residue range 4305-4325 (Fig. 3b).

\section{Non-structural protein 11 (NSP11)}

The cleavage of pp1a polyprotein by $3 \mathrm{CL}^{\text {pro }}$ results in the production of NSP11, a small product whose exact function has not been characterized yet. Depending on the coronavirus species, its length may vary from 13 to 23 amino acid residues [82]. In the case of SARS-CoV-2, the sequence of NSP11 is: SADAQSFLNGFAV [97]. The independent function of NSP11 is not known yet; however, NSP11 becomes the N-terminal of NSP12 during ribosomal frameshift of ORF1b required for NSP11-NSP16 translation [98]. Gadhave and colleagues suggested the helical propensity of NSP11, as studied through SDS micelle experiments [99]. The intrinsic disorder prediction was not performed for NSP11 due to the short sequence.

\section{Non-structural protein 12 (NSP12)/RdRp}

NSP12 is one of the most important proteins required for viral growth. It is associated with both replication and transcription of coronaviruses. This protein is $\sim 932$ amino acid residues in length and is generally referred to as RNAdependent RNA polymerase ( $\mathrm{RdRp}$ ) [82]. RdRp proteins contain multiple domains, which catalyzes the production of phosphodiester bonds between the ribonucleotides in the presence of a divalent metal ion [100]. NSP12 of SARSCoV-2 possesses more than $95 \%$ similarity to polymerases of SARS-CoV. It is inhibited by Remdesivir, a nucleoside 
analog that binds to NSP12 for bringing out this inhibition [101]. NSP12 of SARS-CoV-2 exhibited decreased thermal stability and enzyme activity compared to SARS-CoV NSP12 [102].

NSP12 in the presence of cofactors such as NSP7 and NSP8 mediates the replication and transcription of the virus with high efficiency, instead of being independent for mediating the replication function [103]. NSP12-NSP7-NSP8 thus serves as a minimal unit for carrying out the viral replication [102]. NSP7 and NSP8 initiate the primase activity, followed by the facilitation of NSP12 polymerase activity for viral replication [104]. NSP12 interacts with various other proteins or transcription factors, including CREB regulated transcription coactivator 3 , ribonucleoprotein 4B, PLKHA5, E3 ubiquitin ligase, and ubiquitin associated protein 2, for transcription of the virus [105]. The intrinsic disorder graph showed that NSP12 has multiple intrinsically disordered regions distributed throughout the protein (Fig. 3c).

\section{Non-structural protein-13 (NSP13)/helicase}

NSP13 is $\sim 601$ amino acid residue protein that acts as a helicase with specificity for dsDNA and dsRNA as a substrate with 5'-3' polarity [106]. NSP13 exhibits nucleoside triphosphate hydrolase (NTPase) activity for hydrolysis of different nucleosides. It is involved in unwinding the viral genome by binding to a single-stranded template extending from 5' to 3' direction using ATP hydrolysis [107]. Generally, NSP13 is more efficient in unwinding duplex DNA than duplex RNA; however, in higher concentrations of ATP, unwinding of duplex RNA is preferred to exhibit higher processivity than duplex DNA [106, 108]. Since, ATP induces a conformational change in helicase directing its affinity towards RNA. Therefore, it is believed that changing the ATP concentration and availability may alter the unwinding and translocation of helicase from its substrate. NSP13 may lead to helicase dissociation from the substrate at the replication site, thus becoming a promising candidate for developing effective SARS-CoV-2 antiviral strategies [98]. Shu and colleagues have identified inhibition of both NTPase and helicase activity of NSP13 by addition of Bismuth salts in a dose-dependent manner [109].

NSP13 is a multifunctional enzyme involved in inhibiting type1 interferon (IFN) response in addition to showing a helicase activity. It inhibits the activation of IFN- $\beta$ and IFN- $\alpha$ associated signaling in HEK293T cells $[110,111]$. Moreover, NSP13 assists in replication by interacting with NSP12 along with its participation in mRNA capping. The catalytic efficiency of NSP13 is increased by twofolds in association with NSP12 [112]. The intrinsic disorder graph showed that NSP13 has several intrinsically disordered regions distributed throughout the protein (Fig. 4a).

\section{Non-structural protein 14 (NSP14) / Proofreading exoribonuclease}

NSP14, a 527 amino acid protein, stimulates 3' -5 ' exoribonuclease (proofreading) activity in interaction with NSP10 and N7-guanine methyltransferase activity for capping of viral mRNA and its prevention from degradation [82]. An exoribonuclease domain is required for maintaining the capping function [92]. The proofreading ability of NSP14 helps in the excision of any misincorporated nucleotides, thus protecting the viral genomic from mutations. Various studies were performed to judge the potential of NSP14 in viral replication. Mutations in NSP14 of coronavirus murine hepatitis virus were associated with a 15 -fold increase in mutations in the viral genome, suggesting their involvement in maintaining the replication fidelity.

Similarly, the mutations in NSP14 of SARS-CoV showed a 21-fold increase in genomic mutations [113]. NSP14 of SARS-CoV also inhibits the production and signaling of INF- $\beta$ by blocking the IRF3 localization to the nucleus [111]. NSP14 is known for attenuating the therapeutic potential of several antiviral drugs that act through premature termination of viral replication. Narayanan and Nair had shown binding of ritonavir drug to the active site, i.e., exoribonuclease domain of NSP14, as a probable therapeutic agent for SARS-CoV-2 and its ability to reverse the inhibitory effects on various other drugs of therapeutic potential against coronaviruses [114].

NSP14 can interact with other NSPs, including NSP7, NSP8, NSP10, and NSP12 [115]. When associated with the NSP7-NSP8-NSP12 super-complex, exoribonuclease activity is not shown by NSP14 unless NSP10 associates with the replication complex [103]. This association of all NSPs like $7,8,10,12$, and 14 leads to the formation of another supercomplex with proteins possessing primase, polymerase, and exoribonuclease activities. The intrinsic disorder graph showed that NSP14 has an intrinsically disordered region at the N-terminal tail spanning from 6040 to 6050 (Fig. 4b).

\section{Non-structural protein 15 (NSP15)/Uridylate-specific endoribonuclease}

NSP15 is made up of $~ 346$ amino acid residues in length. This protein possesses multifunctional roles, including an inhibitor of IFN, a binding partner for retinoblastoma protein $(\mathrm{pRb})$, endoribonuclease activity, and helps prevent the virus from dsRNA sensors in the host [116]. Firstly, this protein mediates the cleavage of RNA nucleotides in the $3^{\prime}$ direction of uridylates, hence behaving as an endoribonuclease [117]. Second, much like NSP13 and NSP14, NSP15 can also block the nuclear localization of IRF3 and could thus inhibit the IFN production during viral infection [118]. Third, NSP15 possesses a domain that has a binding affinity towards $\mathrm{pRb}$. 
In the Huh-7 cells, NSP15 can increase the cytoplasmic to a nuclear ratio of $\mathrm{pRb}$ by changing its distribution pattern. Such changes in the form of reduced expression of $\mathrm{Rb}$ cause a change in the cell cycle progression in NSP15-transfected cells [119]. Moreover, it has been observed that magnesium ions can alter the conformation of NSP15 [120], and mutation within the active site or outside the active site of NSP15 can result in the inhibition of viral infection rates [121]. The intrinsic disorder analysis suggested that NSP15 has a few disordered regions spanning throughout the protein (Fig. 4c).

\section{Non-structural protein 16 (NSP16)/2'-0-methyltransferase}

NSP16 is composed of $~ 298$ amino acid residues in length. NSP16, upon interaction with NSP10, gets activated and facilitates the viral mRNA capping as it possesses the 2'-O-MTase domain [82]. NSP16 is an S-adenosylmethionine dependent-methyltransferases required for the virus' life cycle, where NSP10 acts as an essential cofactor for regulating the enzyme activities of NSP16 [122]. Studies on different cell cultures show MTase activity is essential for the replication of coronaviruses. It may also help the virus escape the host's antiviral sensors and thus successfully initiate the viral infection [123, 124]. Many significant studies have been performed on investigating the structure of NSP16 in complex with NSP10; however, more studies on the individual structure of NSP16 are warranted in the future [125]. The intrinsic disorders analysis suggested that NSP16 has one major disordered region spanning at the C-terminal region of the protein (Fig. 4d).

\section{Conclusions}

The viral genome encodes several proteins that are not sufficient to support viral replication.Thus, viruses dependent on the host machinery to complete their life cycles. Usually, viruses have extremely efficient genomes. If a viral protein comprises intrinsic disorders regions, it can be implicated in various functions because it can interacts with different partner proteins. This mechanism is common in the SARSCoV-2 genome that encodes 16 NSPs, which play numerous roles in virus replication and assembly. They contribute to viral pathogenesis by regulating early transcription, gene transactivation, helicase activity, and immunomodulation. Like other viral proteomes, SARS-CoV-2 has a dark proteome where all the proteins, especially NSPs, have a chunk of intrinsic disorders. Exploring the intrinsic disorders in the SARS-CoV-2 NSPs is vital to understanding the virulence of the virus and its subsequent infection.

In this study, we performed a sequence-based analysis of SARS-CoV-2 NSPs to get insights into their intrinsic disorders and their possible functions in the viral replication. The available sequence data for SARS-CoV-2 NSPs were analyzed to evaluate their intrinsic disorders by considering their biological activity relationships. The analysis showed that SARS-CoV-2 NSPs have many intrinsic disorder regions which might lack a well-defined tertiary structure in native conditions. In conclusion, intrinsic disorders are of use to SARS-CoV-2 as they allow efficient usage of the replication machinery, enable it to tolerate the high mutation frequency and changing environments. As such, targeting the intrinsic disorders conferred to the SARS-CoV-2 NSPs to impair critical protein-protein interactions could establish a broad and tempting antiviral policy in COVID-19 research.

Acknowledgements This work was supported by Taif University Researchers Supporting Project Number (TURSP-2020/131), Taif University, Taif, Saudi Arabia. MIH acknowledges the Council of Scientific and Industrial Research for financial support [Project No. 27(0368)/20/EMR-II] for financial support. The authors sincerely thank the Department of Science and Technology, Government of India, for the FIST support (FIST program No. SR/FST/LSII-782/2020).

Author contributions FA, AS and MIH contributed to the conception and design of this study. TM, VNU, AS, PA and DKY performed the experiments and analyzed the data. FA, VNU, SS, TM and MIH interpreted the results of the experiments. TM, AS, PA, DKY and AI wrote the manuscript. VNU, TM, AS, FA, PA and MIH edited and revised the manuscript. All authors approved the final version of the manuscript submitted for publication.

Funding This work was supported by Taif University Researchers Supporting Project Number (TURSP-2020/131), Taif University, Taif, Saudi Arabia and the Indian Council of Medical Research (Grant No. ISRM/12(22)/2020).

Data availability All data generated or analyzed during this study are included to this article.

\section{Declarations}

Conflict of interest The authors declare no conflict of interest.

Ethical approval for Human Subject Not applicable.

Informed consent Not applicable.

\section{References}

1. Mungroo MR, Khan NA, Siddiqui R (2020) Novel coronavirus: current understanding of clinical features, diagnosis, pathogenesis, and treatment options. Pathogens 9:297

2. Wan Y, Shang J, Graham R, Baric RS, Li F (2020) Receptor recognition by the novel coronavirus from Wuhan: an analysis based on decade-long structural studies of SARS coronavirus. J Virol 94

3. Organization WH: WHO coronavirus disease (COVID-19) dashboard. 2020. In: 2020 
4. Iftimie S, López-Azcona AF, Vallverdú I, Hernández-Flix S, De Febrer G, Parra S, Hernández-Aguilera A, Riu F, Joven J, Andreychuk N (2021) First and second waves of coronavirus disease-19: a comparative study in hospitalized patients in Reus, Spain. PLoS ONE 16:e0248029

5. de Castro F (2020) Modelling of the second (and subsequent) waves of the coronavirus epidemic. Spain and Germany as case studies. medRxiv

6. Boroujeni M, Saberian M, Li J (2021) Environmental impacts of COVID-19 on Victoria, Australia, witnessed two waves of Coronavirus. Environ Sci Pollut Res 28:14182-14191

7. Lai JW, Cheong KH (2020) Superposition of COVID-19 waves, anticipating a sustained wave, and lessons for the future. BioEssays 42:2000178

8. Asrani P, Eapen MS, Hassan MI, Sohal SS (2021) Implications of the second wave of COVID-19 in India. Lancet Respir Med 9(9):e93-e94. https://doi.org/10.1016/S2213-2600(21)00312-X

9. Atalan A (2020) Is the lockdown important to prevent the COVID-19 pandemic? Effects on psychology, environment and economy-perspective. Ann Med Surg 56:38-42

10. Asrani P, Hassan MI (2021) SARS-CoV-2 mediated lung inflammatory responses in host: targeting the cytokine storm for therapeutic interventions. Mol Cell Biochem 476:675-687

11. Asrani P, Hassan MI (2020) SARS-CoV-2 mediated lung inflammatory responses in host: targeting the cytokine storm for therapeutic interventions. Mol Cell Biochem

12. Asrani P, Eapen MS, Chia C, Haug G, Weber HC, Hassan MI, Sohal SS (2021) Diagnostic approaches in COVID-19: clinical updates. Expert Rev Respir Med 15:197-212

13. Asrani P, Hussain A, Nasreen K, Alajmi MF, Amir S, Sohal SS, Hassan MI (2021) Guidelines and safety considerations in the laboratory diagnosis of sars-cov-2 infection: a prerequisite study for health professionals. Risk Manage Healthcare Policy 14:379-389

14. Kandeel M, Al-Taher A, Li H, Schwingenschlogl U, Al-Nazawi M (2018) Molecular dynamics of Middle East respiratory syndrome coronavirus (MERS CoV) fusion heptad repeat trimers. Comput Biol Chem 75:205-212

15. Neuman BW, Kiss G, Kunding AH, Bhella D, Baksh MF, Connelly S, Droese B, Klaus JP, Makino S, Sawicki SG (2011) A structural analysis of $\mathrm{M}$ protein in coronavirus assembly and morphology. J Struct Biol 174:11-22

16. Risco C, Antón IM, Enjuanes L, Carrascosa JL (1996) The transmissible gastroenteritis coronavirus contains a spherical core shell consisting of M and N proteins. J Virol 70:4773-4777

17. Ruch TR, Machamer CE (2012) The coronavirus E protein: assembly and beyond. Viruses 4:363-382

18. Malik YA (2020) Properties of coronavirus and SARS-CoV-2. Malays J Pathol 42:3-11

19. Asrani P, Hasan GM, Sohal SS, Hassan MI (2020) Molecular basis of pathogenesis of coronaviruses: a comparative genomics approach to planetary health to prevent zoonotic outbreaks in the 21st century. OMICS J Integrat Biol 24:634-644

20. Asrani P, Tiwari K, Eapen MS, McAlinden KD, Haug G, Johansen MD, Hansbro PM, Flanagan KL, Hassan MI, Sohal SS (2022) Clinical features and mechanistic insights into drug repurposing for combating COVID-19. Int J Biochem Cell Biol $142: 5$

21. Mohammad T, Choudhury A, Habib I, Asrani P, Mathur Y, Umair M, Anjum F, Shafie A, Yadav DK, Hassan MI (2021) Genomic variations in the structural proteins of SARS-CoV-2 and their deleterious impact on pathogenesis: a comparative genomics approach. Front Cell Infect Microbiol 11

22. Müller C, Schulte FW, Lange-Grünweller K, Obermann W, Madhugiri R, Pleschka S, Ziebuhr J, Hartmann RK, Grünweller A
(2018) Broad-spectrum antiviral activity of the eIF4A inhibitor silvestrol against corona-and picornaviruses. Antiviral Res 150:123-129

23. Tang C, Deng Z, Li X, Yang M, Tian Z, Chen Z, Wang G, Wu W (2020) Feng W-h and Zhang G: Helicase of type 2 porcine reproductive and respiratory syndrome virus strain $\mathrm{HV}$ reveals a unique structure. Viruses 12:215

24. Alsaadi JEA, Jones IM (2019) Membrane binding proteins of coronaviruses. Future Virol 14:275-286

25. Naqvi AAT, Fatima K, Mohammad T, Fatima U, Singh IK, Singh A, Atif SM, Hariprasad G, Hasan GM and Hassan MI (2020) Insights into SARS-CoV-2 genome, structure, evolution, pathogenesis and therapies: Structural genomics approach. Biochimica et Biophysica Acta (BBA)-Molecular Basis of Disease 1866: 165878

26. Consortium U (2019) UniProt: a worldwide hub of protein knowledge. Nucleic Acids Res 47:D506-D515

27. Sayers EW, Cavanaugh M, Clark K, Ostell J, Pruitt KD, KarschMizrachi I (2019) GenBank. Nucleic Acids Res 47:D94-D99

28. Bomma R, Venkatesh P, Kumar A, Babu A, Rao S (2012) PONDR (predicators of natural disorder regions). Int J Compt Technol Electron Eng 21:61-70

29. Mészáros B, Erdôs G, Dosztányi Z (2018) IUPred2A: contextdependent prediction of protein disorder as a function of redox state and protein binding. Nucleic Acids Res 46:W329-W337

30. Xue B, Blocquel D, Habchi J, Uversky AV, Kurgan L, Uversky VN, Longhi S (2014) Structural disorder in viral proteins. Chem Rev 114:6880-6911

31. da Silva SJR, da Alves Silva CT, MendePena RPGL (2020) Role of nonstructural proteins in the pathogenesis of SARS-CoV-2. J Med Virol 92:1427-1429

32. Ziebuhr J (2005) The coronavirus replicase. Coronavirus replication and reverse genetics 57-94

33. Huang C, Lokugamage KG, Rozovics JM, Narayanan K, Semler BL, Makino S (2011) SARS coronavirus nsp1 protein induces template-dependent endonucleolytic cleavage of mRNAs: viral mRNAs are resistant to nsp1-induced RNA cleavage. PLoS Pathog 7:e1002433

34. Lokugamage KG, Narayanan K, Huang C, Makino S (2012) Severe acute respiratory syndrome coronavirus protein nsp1 is a novel eukaryotic translation inhibitor that represses multiple steps of translation initiation. J Virol 86:13598

35. Narayanan K, Ramirez SI, Lokugamage KG, Makino S (2015) Coronavirus nonstructural protein 1: common and distinct functions in the regulation of host and viral gene expression. Virus Res 202:89-100

36. Kamitani W, Huang C, Narayanan K, Lokugamage KG, Makino S (2009) A two-pronged strategy to suppress host protein synthesis by SARS coronavirus Nsp1 protein. Nat Struct Mol Biol 16:1134

37. Tanaka T, Kamitani W, DeDiego ML, Enjuanes L, Matsuura Y (2012) Severe acute respiratory syndrome coronavirus nsp1 facilitates efficient propagation in cells through a specific translational shutoff of host mRNA. J Virol 86:11128

38. Thoms M, Buschauer R, Ameismeier M, Koepke L, Denk T, Hirschenberger M, Kratzat H, Hayn M, Mackens-Kiani T, Cheng J (2020) Structural basis for translational shutdown and immune evasion by the Nsp1 protein of SARS-CoV-2. Science 369:1249-1255

39. Schubert K, Karousis ED, Jomaa A, Scaiola A, Echeverria B, Gurzeler L-A, Leibundgut M, Thiel V, Mühlemann O, Ban N (2020) SARS-CoV-2 Nsp1 binds the ribosomal mRNA channel to inhibit translation. Nat Struct Mol Biol 27:959-966

40. Brockway SM, Denison MR (2005) Mutagenesis of the murine hepatitis virus nsp1-coding region identifies residues important 
for protein processing, viral RNA synthesis, and viral replication. Virology 340:209-223

41. Galán C, Enjuanes L, Almazán F (2005) A point mutation within the replicase gene differentially affects coronavirus genome versus minigenome replication. J Virol 79:15016

42. Clark LK, Green TJ, Petit CM (2021) Structure of nonstructural protein 1 from SARS-CoV-2. J Virol 95

43. Khan S, Fakhar Z, Hussain A, Ahmad A, Jairajpuri DS, Alajmi MF, Hassan MI (2020) Structure-based identification of potential SARS-CoV-2 main protease inhibitors. J Biomol Struct Dynam

44. Shamsi A, Mohammad T, Anwar S, AlAjmi MF, Hussain A, Md. Tabish R, Islam A, Md. Imtaiyaz H (2020) Glecaprevir and Maraviroc are high-affinity inhibitors of SARS-CoV-2 main protease: Possible implication in COVID-19 therapy. Bioscience reports 40

45. Jairajpuri DS, Hussain A, Nasreen K, Mohammad T, Anjum F, Rehman MT, Hasan GM, Alajmi MF, Hassan MI (2021) Identification of natural compounds as potent inhibitors of SARS-CoV-2 main protease using combined docking and molecular dynamics simulations. Saudi J Biol Sci 28:2423-2431

46. Qayyum S, Mohammad T, Slominski RM, Hassan MI, Tuckey R, Raman C, Slominski AT (2021) Vitamin D and lumisterol novel metabolites can inhibit SARS-COV-2 replication machinery enzymes. Am J Physiol-Endocrinol Metabol

47. Shamsi A, Mohammad T, Anwar S, Amani S, Khan MS, Husain FM, Rehman MT, Islam A, Hassan MI (2021) Potential drug targets of SARS-CoV-2: from genomics to therapeutics. Int $\mathbf{J}$ Biol Macromol 177:1-9

48. Davies JP, Almasy KM, McDonald EF, Plate L (2020) Comparative multiplexed interactomics of SARS-CoV-2 and homologous coronavirus nonstructural proteins identifies unique and shared host-cell dependencies. ACS Infect Dis

49. Angeletti S, Benvenuto D, Bianchi M, Giovanetti M, Pascarella S, Ciccozzi M (2020) COVID-2019: the role of the nsp2 and nsp3 in its pathogenesis. J Med Virol 92:584-588

50. Cornillez-Ty CT, Liao L, Yates JR III, Kuhn P, Buchmeier MJ (2009) Severe acute respiratory syndrome coronavirus nonstructural protein 2 interacts with a host protein complex involved in mitochondrial biogenesis and intracellular signaling. J Virol 83:10314-10318

51. Graham RL, Sims AC, Baric RS, Denison MR (2006) The nsp2 proteins of mouse hepatitis virus and SARS coronavirus are dispensable for viral replication. In: The Nidoviruses. Springer, pp 67-72

52. Snijder EJ, Bredenbeek PJ, Dobbe JC, Thiel V, Ziebuhr J, Poon LL, Guan Y, Rozanov M, Spaan WJ, Gorbalenya AE (2003) Unique and conserved features of genome and proteome of SARS-coronavirus, an early split-off from the coronavirus group 2 lineage. J Mol Biol 331:991-1004

53. Lu J-H, Zhang D-M, Wang G-L, Guo Z-M, Li J, Tan B-Y, OuYang L-P, Ling W-H, Yu X-B, Zhong N-S (2005) Sequence analysis and structural prediction of the severe acute respiratory syndrome coronavirus nsp5. Acta Biochim Biophys Sin $37: 473-479$

54. Anand K, Ziebuhr J, Wadhwani P, Mesters JR, Hilgenfeld R (2003) Coronavirus main proteinase (3CLpro) structure: basis for design of anti-SARS drugs. Science 300:1763-1767

55. Báez-Santos YM, John SES, Mesecar AD (2015) The SARScoronavirus papain-like protease: structure, function and inhibition by designed antiviral compounds. Antiviral Res 115:21-38

56. Sakai Y, Kawachi K, Terada Y, Omori H, Matsuura Y, Kamitani W (2017) Two-amino acids change in the nsp4 of SARS coronavirus abolishes viral replication. Virology 510:165-174

57. Tan J, Vonrhein C, Smart OS, Bricogne G, Bollati M, Kusov Y, Hansen G, Mesters JR, Schmidt CL, Hilgenfeld R (2009) The
SARS-unique domain (SUD) of SARS coronavirus contains two macrodomains that bind G-quadruplexes. PLoS Pathog 5:e1000428

58. Johnson MA, Chatterjee A, Neuman BW, Wüthrich K (2010) SARS coronavirus unique domain: three-domain molecular architecture in solution and RNA binding. J Mol Biol 400:724-742

59. Neuman BW, Joseph JS, Saikatendu KS, Serrano P, Chatterjee A, Johnson MA, Liao L, Klaus JP, Yates JR III, Wüthrich K (2008) Proteomics analysis unravels the functional repertoire of coronavirus nonstructural protein 3. J Virol 82:5279-5294

60. Serrano P, Johnson MA, Almeida MS, Horst R, Herrmann T, Joseph JS, Neuman BW, Subramanian V, Saikatendu KS, Buchmeier MJ (2007) Nuclear magnetic resonance structure of the $\mathrm{N}$-terminal domain of nonstructural protein 3 from the severe acute respiratory syndrome coronavirus. J Virol 81:12049

61. Oostra M, Te Lintelo E, Deijs M, Verheije M, Rottier P, De Haan C (2007) Localization and membrane topology of coronavirus nonstructural protein 4 : involvement of the early secretory pathway in replication. J Virol 81:12323-12336

62. Yu D, Rao S, Tsai LM, Lee SK, He Y, Sutcliffe EL, Srivastava M, Linterman M, Zheng L, Simpson N (2009) The transcriptional repressor Bcl-6 directs $\mathrm{T}$ follicular helper cell lineage commitment. Immunity 31:457-468

63. Fenech EJ, Lari F, Charles PD, Fischer R, Laétitia-Thézénas M, Bagola K, Paton AW, Paton JC, Gyrd-Hansen M, Kessler BM (2020) Interaction mapping of endoplasmic reticulum ubiquitin ligases identifies modulators of innate immune signalling. Elife 9:e57306

64. Owen RP, Gong L, Sagreiya H, Klein TE, Altman RB (2010) VKORC1 pharmacogenomics summary. Pharmacogenet Genom 20:642

65. Wu C, Liu Y, Yang Y, Zhang P, Zhong W, Wang Y, Wang Q, Xu Y, Li M, Li X (2020) Analysis of therapeutic targets for SARSCoV-2 and discovery of potential drugs by computational methods. Acta Pharmaceutica Sinica B 10:766-788

66. Stobart CC, Sexton NR, Munjal H, Lu X, Molland KL, Tomar S, Mesecar AD, Denison MR (2013) Chimeric exchange of coronavirus nsp5 proteases (3CLpro) identifies common and divergent regulatory determinants of protease activity. J Virol 87:12611-12618

67. Yang H, Yang M, Ding Y, Liu Y, Lou Z, Zhou Z, Sun L, Mo L, Ye S, Pang H (2003) The crystal structures of severe acute respiratory syndrome virus main protease and its complex with an inhibitor. Proc Natl Acad Sci 100:13190-13195

68. Raj R (2021) Analysis of non-structural proteins, NSPs of SARSCoV-2 as targets for computational drug designing. Biochem Biophys Rep 25:100847

69. Angelini MM, Akhlaghpour M, Neuman BW, Buchmeier MJ (2013) Severe acute respiratory syndrome coronavirus nonstructural proteins 3,4 , and 6 induce double-membrane vesicles. MBio 4:e00524-e613

70. Santerre M, Arjona SP, Allen CN, Shcherbik N, Sawaya BE (2020) Why do SARS-CoV-2 NSPs rush to the ER? J Neurol

71. Oostra M, Hagemeijer MC, Van Gent M, Bekker CP, Te Lintelo EG, Rottier PJ, De Haan CA (2008) Topology and membrane anchoring of the coronavirus replication complex: not all hydrophobic domains of nsp3 and nsp6 are membrane spanning. J Virol 82:12392-12405

72. Baliji S, Cammer SA, Sobral B, Baker SC (2009) Detection of nonstructural protein 6 in murine coronavirus-infected cells and analysis of the transmembrane topology by using bioinformatics and molecular approaches. J Virol 83:6957-6962

73. Benvenuto D, Angeletti S, Giovanetti M, Bianchi M, Pascarella S, Cauda R, Ciccozzi M, Cassone A (2020) Evolutionary 
analysis of SARS-CoV-2: how mutation of Non-Structural Protein 6 (NSP6) could affect viral autophagy. J Infect 81:e24-e27

74. Forni D, Cagliani R, Clerici M, Sironi M (2017) Molecular evolution of human coronavirus genomes. Trends Microbiol 25:35-48

75. Zhou A, Li S, Khan FA, Zhang S (2016) Autophagy postpones apoptotic cell death in PRRSV infection through Bad-Beclin1 interaction. Virulence 7:98-109

76. Cottam EM, Whelband MC, Wileman T (2014) Coronavirus NSP6 restricts autophagosome expansion. Autophagy 10:1426-1441

77. Tang JW, Cheung JL, Chu IM, Sung JJ, Peiris M, Chan PK (2006) The large 386-nt deletion in SARS-associated coronavirus: evidence for quasispecies? J Infect Dis 194:808-813

78. Gordon DE, Jang GM, Bouhaddou M, Xu J, Obernier K, White KM, O'Meara MJ, Rezelj VV, Guo JZ, Swaney DL (2020) A SARS-CoV-2 protein interaction map reveals targets for drug repurposing. Nature 583:459-468

79. Kirchdoerfer RN, Ward AB (2019) Structure of the SARSCoV nsp12 polymerase bound to nsp7 and nsp8 co-factors. Nat Commun 10:1-9

80. Gao Y, Yan L, Huang Y, Liu F, Zhao Y, Cao L, Wang T, Sun Q, Ming Z, Zhang L (2020) Structure of the RNA-dependent RNA polymerase from COVID-19 virus. Science 368:779-782

81. Reshamwala SM, Likhite V, Degani MS, Deb SS, Noronha SB. Mutations in SARS-CoV-2 nsp7 and nsp8 proteins and their predicted impact on replication/transcription complex structure. J Med Virol

82. Snijder E, Decroly E, Ziebuhr J (2016) The nonstructural proteins directing coronavirus RNA synthesis and processing. Adv Virus Res 96:59-126

83. Tan YW, Fung TS, Shen H, Huang M, Liu DX (2018) Coronavirus infectious bronchitis virus non-structural proteins 8 and 12 form stable complex independent of the non-translated regions of viral RNA and other viral proteins. Virology 513:75-84

84. Miknis ZJ, Donaldson EF, Umland TC, Rimmer RA, Baric RS, Schultz LW (2009) Severe acute respiratory syndrome coronavirus nsp9 dimerization is essential for efficient viral growth. J Virol 83:3007-3018

85. Ponnusamy R, Moll R, Weimar T, Mesters JR, Hilgenfeld R (2008) Variable oligomerization modes in coronavirus nonstructural protein 9. J Mol Biol 383:1081-1096

86. Sutton G, Fry E, Carter L, Sainsbury S, Walter T, Nettleship J, Berrow N, Owens R, Gilbert R, Davidson A (2004) The nsp9 replicase protein of SARS-coronavirus, structure and functional insights. Structure 12:341-353

87. Hu T, Chen C, Li H, Dou Y, Zhou M, Lu D, Zong Q, Li Y, Yang C, Zhong Z (2017) Structural basis for dimerization and RNA binding of avian infectious bronchitis virus nsp9. Protein Sci 26:1037-1048

88. Zhang C, Chen Y, Li L, Yang Y, He J, Chen C, Su D (2020) Structural basis for the multimerization of nonstructural protein nsp9 from SARS-CoV-2. Mol Biomed 1:1-9

89. Theobald DL, Mitton-Fry RM, Wuttke DS (2003) Nucleic acid recognition by OB-fold proteins. Annu Rev Biophys Biomol Struct 32:115-133

90. Egloff M-P, Ferron F, Campanacci V, Longhi S, Rancurel C, Dutartre H, Snijder EJ, Gorbalenya AE, Cambillau C, Canard B (2004) The severe acute respiratory syndrome-coronavirus replicative protein nsp9 is a single-stranded RNA-binding subunit unique in the RNA virus world. Proc Natl Acad Sci 101:3792-3796

91. Farias AB, Candiotto G, Siragusa L, Goracci L, Cruciani G, Oliveira ER, Horta BA (2021) Targeting Nsp9 as an anti-SARSCoV-2 strategy. New J Chem 45:522-525
92. Chen Y, Cai H, Xiang N, Tien P, Ahola T, Guo D (2009) Functional screen reveals SARS coronavirus nonstructural protein nsp14 as a novel cap N7 methyltransferase. Proc Natl Acad Sci 106:3484-3489

93. Bouvet M, Debarnot C, Imbert I, Selisko B, Snijder EJ, Canard B, Decroly E (2010) In vitro reconstitution of SARS-coronavirus mRNA cap methylation. PLoS Pathog 6:e1000863

94. Chen Y, Guo D (2016) Molecular mechanisms of coronavirus RNA capping and methylation. Virologica Sinica 31:3-11

95. Rogstam A, Nyblom M, Christensen S, Sele C, Talibov VO, Lindvall T, Rasmussen AA, André I, Fisher Z, Knecht W (2020) Crystal Structure of Non-Structural Protein 10 from Severe Acute Respiratory Syndrome Coronavirus-2. Int J Mol Sci 21:7375

96. Cassandri M, Smirnov A, Novelli F, Pitolli C, Agostini M, Malewicz M, Melino G, Raschellà G (2017) Zinc-finger proteins in health and disease. Cell Death Discov 3:1-12

97. Wu F, Zhao S, Yu B, Chen Y-M, Wang W, Song Z-G, Hu Y, Tao Z-W, Tian J-H, Pei Y-Y (2020) A new coronavirus associated with human respiratory disease in China. Nature 579:265-269

98. Suryawanshi RK, Koganti R, Agelidis A, Patil CD, Shukla D (2020) Dysregulation of cell signaling by SARS-CoV-2. Trends Microbiol

99. Gadhave K, Kumar P, Kumar A, Bhardwaj T, Garg N, Giri R (2021) Conformational dynamics of NSP11 peptide of SARS$\mathrm{CoV}-2$ under membrane mimetics and different solvent conditions. BioRxiv

100. Jia H, Gong P (2019) A structure-function diversity survey of the RNA-dependent RNA polymerases from the positive-strand RNA viruses. Front Microbiol 10:1945

101. Mirza MU, Froeyen M (2020) Structural elucidation of SARSCoV-2 vital proteins: computational methods reveal potential drug candidates against main protease, Nsp12 polymerase and Nsp13 helicase. J Pharmac Anal 10:320-328

102. Peng Q, Peng R, Yuan B, Zhao J, Wang M, Wang X, Wang Q, Sun Y, Fan Z, Qi J (2020) Structural and biochemical characterization of the nsp12-nsp7-nsp8 core polymerase complex from SARS-CoV-2. Cell Rep 31:107774

103. Subissi L, Posthuma CC, Collet A, Zevenhoven-Dobbe JC, Gorbalenya AE, Decroly E, Snijder EJ, Canard B, Imbert I (2014) One severe acute respiratory syndrome coronavirus protein complex integrates processive RNA polymerase and exonuclease activities. Proc Natl Acad Sci 111:E3900-E3909

104. Hillen HS, Kokic G, Farnung L, Dienemann C, Tegunov D, Cramer P (2020) Structure of replicating SARS-CoV-2 polymerase. Nature 584:154-156

105. Bouhaddou M, Memon D, Meyer B, White KM, Rezelj VV, Marrero MC, Polacco BJ, Melnyk JE, Ulferts S, Kaake RM (2020) The global phosphorylation landscape of SARS-CoV-2 infection. Cell 182: 685-712. e19

106. Jang K-J, Jeong S, Kang DY, Sp N, Yang YM, Kim D-E (2020) A high ATP concentration enhances the cooperative translocation of the SARS coronavirus helicase nsP13 in the unwinding of duplex RNA. Sci Rep 10:1-13

107. Seybert A, Hegyi A, Siddell SG, Ziebuhr J (2000) The human coronavirus $229 \mathrm{E}$ superfamily 1 helicase has RNA and DNA duplex-unwinding activities with $5^{\prime}$-to-3' polarity. RNA 6:1056-1068

108. Tanner JA, Watt RM, Chai Y-B, Lu L-Y, Lin MC, Peiris JM, Poon LL, Kung H-F, Huang J-D (2003) The severe acute respiratory syndrome (SARS) coronavirus NTPase/helicase belongs to a distinct class of $5^{\prime}$ to $3^{\prime}$ viral helicases. J Biol Chem 278:39578-39582

109. Shu T, Huang M, Wu D, Ren Y, Zhang X, Han Y, Mu J, Wang R, Qiu Y, Zhang D-Y (2020) SARS-Coronavirus-2 Nsp13 possesses 
NTPase and RNA helicase activities that can be inhibited by bismuth salts. Virologica Sinica 35:321-329

110. Xia H, Cao Z, Xie X, Zhang X, Chen JY-C, Wang H, Menachery VD, Rajsbaum R, Shi P-Y (2020) Evasion of type I interferon by SARS-CoV-2. Cell Rep 33:108234

111. Lei X, Dong X, Ma R, Wang W, Xiao X, Tian Z, Wang C, Wang Y, Li L, Ren L (2020) Activation and evasion of type I interferon responses by SARS-CoV-2. Nat Commun 11:1-12

112. Adedeji AO, Marchand B, Te Velthuis AJ, Snijder EJ, Weiss S, Eoff RL, Singh K, Sarafianos SG (2012) Mechanism of nucleic acid unwinding by SARS-CoV helicase. PLoS ONE 7:e36521

113. Eckerle LD, Lu X, Sperry SM, Choi L, Denison MR (2007) High fidelity of murine hepatitis virus replication is decreased in nsp14 exoribonuclease mutants. J Virol 81:12135-12144

114. Narayanan N, Nair DT (2021) Ritonavir may inhibit exoribonuclease activity of nsp14 from the SARS-CoV-2 virus and potentiate the activity of chain terminating drugs. Int J Biol Macromol 168:272-278

115. Graham RL, Sparks JS, Eckerle LD, Sims AC, Denison MR (2008) SARS coronavirus replicase proteins in pathogenesis. Virus Res 133:88-100

116. Deng X, Hackbart M, Mettelman RC, O'Brien A, Mielech AM, Yi G, Kao CC, Baker SC (2017) Coronavirus nonstructural protein 15 mediates evasion of dsRNA sensors and limits apoptosis in macrophages. Proc Natl Acad Sci 114:E4251-E4260

117. Bhardwaj K, Guarino L, Kao CC (2004) The severe acute respiratory syndrome coronavirus Nsp15 protein is an endoribonuclease that prefers manganese as a cofactor. J Virol 78:12218-12224

118. Yuen C-K, Lam J-Y, Wong W-M, Mak L-F, Wang X, Chu H, Cai J-P, Jin D-Y, To KK-W, Chan JF-W (2020) SARS-CoV-2 nsp13, nsp14, nsp15 and orf6 function as potent interferon antagonists. Emerg Microbes Infect 9:1418-1428

119. Bhardwaj K, Liu P, Leibowitz JL, Kao CC (2012) The coronavirus endoribonuclease Nsp15 interacts with retinoblastoma tumor suppressor protein. J Virol 86:4294-4304
120. Chen S, Zheng X, Zhu J, Ding R, Jin Y, Zhang W, Yang H, Zheng Y, Li X, Duan G (2020) Extended ORF8 gene region is valuable in the epidemiological investigation of severe acute respiratory syndrome-similar coronavirus. J Infect Dis 222:223-233

121. Kang H, Bhardwaj K, Li Y, Palaninathan S, Sacchettini J, Guarino L, Leibowitz JL, Kao CC (2007) Biochemical and genetic analyses of murine hepatitis virus Nsp15 endoribonuclease. J Virol 81:13587-13597

122. Decroly E, Debarnot C, Ferron F, Bouvet M, Coutard B, Imbert I, Gluais L, Papageorgiou N, Sharff A, Bricogne G (2011) Crystal structure and functional analysis of the SARS-coronavirus RNA cap 2'-O-methyltransferase nsp10/nsp16 complex. PLoS Pathog 7:e1002059

123. Daffis S, Szretter KJ, Schriewer J, Li J, Youn S, Errett J, Lin T-Y, Schneller S, Zust R, Dong H (2010) 2'-O methylation of the viral mRNA cap evades host restriction by IFIT family members. Nature 468:452-456

124. Decroly E, Imbert I, Coutard B, Bouvet M, Selisko B, Alvarez K, Gorbalenya AE, Snijder EJ, Canard B (2008) Coronavirus nonstructural protein 16 is a cap-0 binding enzyme possessing (nucleoside-2' O)-methyltransferase activity. J Virol 82:8071-8084

125. Krafcikova P, Silhan J, Nencka R, Boura E (2020) Structural analysis of the SARS-CoV-2 methyltransferase complex involved in RNA cap creation bound to sinefungin. Nat Commun 11:1-7

Publisher's Note Springer Nature remains neutral with regard to jurisdictional claims in published maps and institutional affiliations. 